\title{
Short-course AIT in a porcine allergic model by enhanced and prolonged allergen presentation using a novel mucoadhesive patch approach
}

\author{
Poul Sørensen ${ }^{1}$, Pavlina Turanek-Knotigova ${ }^{2}$, Josef Masek ${ }^{2}$, Jan Kotoucek ${ }^{2}$, František \\ Hubatka $^{2}$, Pavel Kulich ${ }^{2}$, Daniela Lubasova ${ }^{3}$, Milan Raska ${ }^{2}$, Kees Leenhouts ${ }^{4}$, and Jaroslav \\ Turanek $^{2}$ \\ ${ }^{1}$ Aarhus Universitet \\ ${ }^{2}$ Veterinary Research Institute \\ ${ }^{3}$ Technical University of Liberec \\ ${ }^{4}$ Allero Therapeutics
}

July 8, 2020

\begin{abstract}
Background: To assess an improved version of sublingual AIT performed by applying a novel short-course oromucosal allergen administration strategy in a translational allergic pig model by prolonged and enhanced tolerogenic presentation of intact allergen with a novel mucoadhesive patch technology. Methods: Newborn piglets sensitized intraperitoneally with the common egg allergen ovalbumin (OVA) were sublingually treated five times using a dose ascending administration schedule with nanofiberbased mucoadhesive patches carrying OVA allergen, formulated with immune tolerogenic particles derived from the probiotic bacterium Lactococcus lactis (TBP). Immunologic assessment was conducted by the use of the intradermal test of immediate allergic reactions to OVA. Results: Five weekly treatments with the mucoadhesive patch carrying OVA alone or OVA and TBP resulted in significant desensitisation, being $8 \%$ and $45 \%$ respectively, as measured by intradermal testing. Mucoadhesive patch-based administration of OVA together with TBP resulted in the allergen localisation to submucosa tissue and draining lymph nodes and resulted in better desensitization compared with patch containing OVA allergen alone. Conclusion: A first demonstration of short-course SLIT approach which we term Specific Oromucosal Immunotherapy (SOMIT) has been successfully carried out in a new translational therapeutic porcine model of allergy. The results suggest that an improved short-course SLIT approach is feasible by prolonging allergen delivery using a mucoadhesive patch and proper stimulation of a privileged tolerogenic immune compartment by tolerogenic probiotic bacterial particles.
\end{abstract}

\section{Hosted file}

Sorensen et al, Allergy manuscript FINAL.docx available at https://authorea.com/users/340903/ articles/468034-short-course-ait-in-a-porcine-allergic-model-by-enhanced-and-prolongedallergen-presentation-using-a-novel-mucoadhesive-patch-approach 